\title{
Re-engineering plant gene targeting
}

\author{
Anne B. Britt ${ }^{1}$ and Gregory D. May ${ }^{2}$ \\ ${ }^{1}$ Section of Plant Biology, University of California, Davis, CA 95616, USA \\ ${ }^{2}$ Plant Biology Division, The Samuel Roberts Noble Foundation, Ardmore, OK 73401, USA
}

\begin{abstract}
The genome sequence of Arabidopsis is complete and the genomes of plants representing legumes (Medicago truncatula) and grasses (rice) will soon follow. The rate at which new genes have been discovered has far outstripped the pace at which their function is determined. The greatest hurdle that plant biologists face in assigning gene function and in crop improvement is the lack of efficient and robust technologies to generate gene replacements or targeted gene knockouts. Many of the factors underlying these events remain to be elucidated. This review addresses the current status of plant gene targeting and what is known about the associated plant DNA repair mechanisms.
\end{abstract}

Since the development of plant transformation technologies in the early 1980s, functional characterization of newly discovered genes has occurred in a broad range of plant species. In addition to its application to basic research, the ability to express foreign genes or to attenuate expression levels of endogenous genes has made a huge impact on crop improvement.

Given the tremendous resources being invested in plant genomics, data are being generated at an unprecedented rate. Unfortunately, our understanding of gene function lags far behind. The ability to delete, or specifically alter, chromosomal sequences of interest would greatly enhance our ability to test gene function. To date, targeted gene replacement occurs in higher plants at such low frequency as to render it an unfeasible approach for everyday use by biologists. The ability to generate targeted gene changes in plants would have as great an impact on fundamental research and crop improvement as did the original plant transformation experiments that took place more than two decades ago.

In this review, we discuss methods to disrupt plant gene function and the mechanisms by which they operate, in an attempt to better address questions of gene targeting in plants.

\section{Reverse genetic approaches to plant functional genomics}

The ability to knockout genes or suppress their expression has been a powerful tool for plant biologists. Common approaches include insertional mutagenesis, antisense and co-suppression in transgenics, and virus-induced gene silencing. Collections of random T-DNA or transposable element insertion mutants are currently available to the Arabidopsis research community through a variety of sources. These collections total $>200000$ independently derived T-DNA mutants. The flanking DNAs of many of these insertions have been sequenced, and mutants in specific genes can be identified by BLASTing public databases; other mutants can be identified via PCR [1] (http://signal.salk.edu/ and www.tmri.org). These collections are tremendously useful, but they do not always produce null alleles, and smaller gene targets might not be represented at all.

Homology-directed gene silencing (HDGS) [2] provides another avenue for downregulation of gene function. RNA-directed gene silencing was discovered in transgenic plants in which interactions between similar host genes and transgenes resulted in the reduction of mRNA levels for both loci. This phenomenon occurs throughout most plant species, is similar to gene silencing events in other organisms $[3,4]$ and can be induced at the transcriptional (TGS) or post-transcriptional (PTGS) levels [5]. TGS is associated with hyper-methylation of regulatory regions resulting in a substantial decrease in transcription of the targeted gene while PTGS is associated with cleavage of a double- stranded RNA into small interfering RNAs (siRNA) of 21 to 25 nucleotides [6]. siRNAs, which spread throughout the plant, are thought to direct a nuclease complex that degrades the target mRNA and a methylation complex responsible for the methylation of silenced genes [7]. PTGS can be efficiently induced, at least in somatic tissues, by the expression of dsRNAs and therefore provides a useful method for dominant inhibition of gene function $[8,9]$. The downside of HDGS is its somewhat unpredictable nature because of its lack of controllable tissue specificity.

Plant gene expression can also be suppressed through the infection of virus vectors harboring exon-containing regions of the host gene. This approach, referred to as virus-induced gene silencing (VIGS), has been used to silence a variety of plant genes in multiple plant species. Early examples of VIGS used tobacco mosaic virus-based vectors [10] and potato virus X-based vectors [11] that contained a portion of a phytoene desaturase (PDS) gene. The mechanisms by which VIGS occurs are still being elucidated. However, it is known that certain elements are conserved between the various HDGS phenomena.

The various approaches to RNAi each have their own limitations, including incomplete or partial silencing and a lack of member-specificity within a multigene family. VIGS is highly dependent on finding a suitable viral vector for the recipient host species and, like other forms of PTGS, lacks tissue specificity because of the systemic nature of the silencing signal. Nevertheless, these 
approaches have provided plant biologists with powerful tools to alter gene function.

\section{Transgene insertion via double-strand-break repair: two competing pathways}

In Saccharomyces cerevisiae, transgenes (including T-DNAs $[12,13])$ with homology to the yeast chromosome integrate via homologous recombination ( $\mathrm{HR}$ ), producing gene replacement events. In most vertebrate tissues and in all higher plants, transforming plasmids and T-DNAs integrate via nonhomologous end joining (NHEJ), resulting in random gene addition events [14]. What accounts for this difference? It's conceivable that the plant genome, perhaps owing to its larger size, is an impossibly difficult substrate for gene targeting. Most vertebrate cells, like plant cells, have large genomes and integrate transgenes nonhomologously. But the exceptions to this vertebrate rule prove that genome size does not have to be an impassible barrier to gene replacement. Adult mouse cells ( $\mathrm{C}=3$ gigabases $)$ integrate transgenes nonhomologously, but mouse embryonic stem cells can integrate transgenes via homologous recombination frequently enough to make directed knockouts feasible in this relatively expensive model system [15]. Chicken ( $\mathrm{C}=1.2$ gigabases $)$ DT40 cells integrate transgenes primarily via homologous recombination [16]. In addition, the Physcomitrella genome is three times as large as that of Arabidopsis, and yet gene replacement occurs efficiently in the chloronemal cells of this moss [17-19] (D.G. Schaefer, PhD thesis, University of Lausanne, 1994). Thus, a large genome might or might not be a good target for gene replacement, apparently depending on the pattern of expression of proteins involved in the process, presumably double-strandbreak-repair proteins. Yeasts, plants and vertebrates all possess the ability to repair double-strand breaks (DSBs), or integrate transgenes, via both HR and NHEJ [20-23], but choose to employ these pathways to varying extents. Thus, by altering the pattern of expression of these genes it might be possible to alter the mode of transgene integration.

The relative rarity of transgene replacement events versus nonhomologous events $(\sim 1$ replacement event to 3000 nonhomologous events [24-26]) suggests that substantial reengineering of the DSB repair process will have to be performed. This might be an impossibly complex task, requiring just the right level of expression of a variety of collaborating gene products. More optimistically, one might hope that the enhanced expression of a protein that's currently a bottleneck in the homologous repair pathway, or perhaps the elimination of the competing NHEJ pathway, might enhance the ratio of homologous to nonhomologous events. Many, although not all, of the genes required for both the NHEJ and HR DSB repair pathways have been identified in yeast and also in vertebrates, although the biochemical functions of many of these proteins remains to be determined. The completion of the Arabidopsis genome project has shown that plants carry what seems to be the vertebrate suite of genes required for double-strand-break repair [27]. Screens for ionizing radiation- or bleomycin-sensitive mutants [28-34], which can be defective in DSB repair, as well as assays for the direct identification of plants defective in T-DNA transformation [35-37], have been developed and will continue to be employed to identify potentially novel genes involved in this process. Thus, Arabidopsis appears to have an established infrastructure for the study and manipulation of transgene integration pathways.

\section{Transgene integration via HR: possible targets for upregulation}

There are many models, and perhaps just as many pathways, for the repair of DSBs via homologous recombination. For further guidance, see Ref. [38] for a discussion of models for gene replacement. In Fig. 1, we present two different mechanisms for homologous recombinational repair of DSBs and list the proteins involved in these processes. The essential difference between the two mechanisms is the establishment of a Holliday junction in the double crossover (Szostak) model; this presents an

(a) Resection of double-strand break

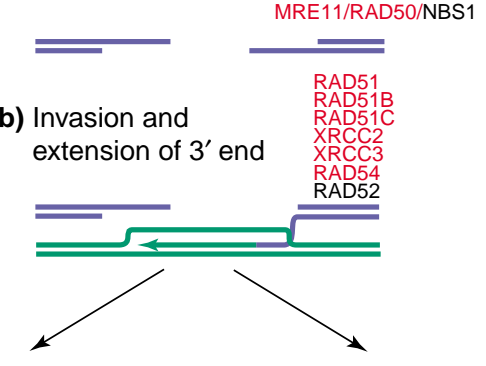

Double-crossover mechanism

Synthesis-dependent strand annealing (c)

Establishment of double-

Holliday junction and DNA synthesis

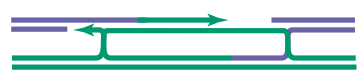

(d)

Ligation and branch

migration

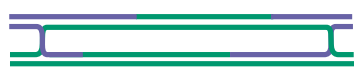

(e)

Resolution (here as a

non-crossover) and ligation MUS81/EME1

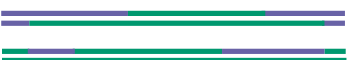

(c)

Collapse of D-loop, reannealing to broken chromatid

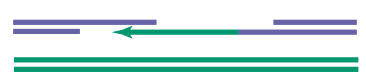

(d)

DNA synthesis and ligation

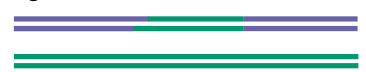

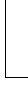

Fig. 1. Double-strand break repair via homologous recombination. Arabidopsis homologs thought to be involved, based on mammalian models [51] are written in red. Proteins written in black are required for homologous recombination (HR) in some other organisms but obvious homologs do not exist in plants. These include NBS1 (in S. cerevisiae, the distantly related protein XRS2), EME1 (in Schizosaccharomyces pombe, with no obvious sequence homolog in mammals), and RAD52 (crucial for most HR events in yeasts, but less so in mammals $[63,64]$ ). The eukaryotic genes required for branch migration have not yet been identified, although a mammalian branch migration activity (with an associated resolvase unrelated to MUS81) has been biochemically characterized [75]. Arabidopsis mutants defective in RAD50 are viable and have been characterized, and evidence suggests a role in the maintenance of genomic integrity $[76,77]$ and meiotic recombination [78]. Arrowheads represent $3^{\prime}$ ends; the invading (damaged, or transgene) strands are depicted in purple; the template strands are in green. 
opportunity for chromosome arm exchange. Although these exchanges are required for correct segregation of homologs during meiosis, they might be hazardous during repair of DSBs on nonaligned chromosomes or chromatids; exchange between dispersed repetitive sequences might generate deletions, duplications or translocations. However, the synthesis-dependent strand-annealing (SDSA, Fig. 1) mechanism eliminates the need for a Holliday junction and the hazards associated with its resolution. Evidence points to SDSA as a major pathway in plants for the repair of chromosomal double-strand breaks induced by restriction enzymes [39] or transposable elements [40].

How might these DSB repair pathways be employed for the integration of transgenes via HR? Presumably the free ends are found on the transgene (rather than at the recipient locus) and these must be either spliced into (Szostak model) or somehow copied onto (SDSA) the target. It is possible to develop SDSA models for the integration of transgenes; these models require either the break-induced replication (BIR) of the entire chromosome (a phenomenon known to occur in yeast [38]) or the encounter of a nick on the displaced strand, with subsequent trimming of flaps. An SDSA model can also explain the integration of 'ends-in' constructs such as those employed in the Drosophila gene-targeting system [41]. The Szostak model might explain the integration of 'ends out' constructs (Fig. 2).

Homologous transgene integration via the Szostak model requires the induction of nicks in the target site. These might be generated by Holliday junction resolvases, by a flap endonuclease, or through replication to a preexisting nick. Enzymes specifically required for the generation of target-site nicks during gene replacement have recently been identified in mice. Using $m E r c c 1^{-1-}$ embryonic stem cell knockouts, it has been shown [42] that the structure-specific $3^{\prime}$ flap endonuclease [43] encoded by $m E r c c 1$ and $m X P F$ is required for gene replacement. The complex is involved in the integration of gene replacement constructs that involve the insertion of a significant piece of heterologous sequence (such as a selectable marker) within the target gene (Fig. 2). It might also be involved in the trimming of nonhomologous flanking ends [44]. Homologs of both ERCC1 and XPF have been identified in the Arabidopsis database (The Arabidopsis Genome Initiative, 2000), and mutants in both genes identified $[33,45,46]$; the mutants are gamma-sensitive, UV sensitive, and sensitive to the crosslinking agent mitomycin C. The role of $U V H 1$ (the Arabidopsis XPF homolog) in nontargeted T-DNA integration has been investigated, with conflicting results [47-49]. The requirement for this endonuclease in gene replacement in plants has yet to be investigated.

Attempts have been made to stimulate the HR pathway in plants, with the goal of improving the efficiency of gene replacement. These include the overexpression of $E$. coli RecA [24,50] and the expression of RuvC Holliday junction resolvase proteins [51]. Both efforts have resulted in an increase in spontaneous homologous recombination, which might reflect either an increase in the efficiency of $\mathrm{HR}$ or an increase in the rate of induction of recombinogenic DNA damage. The (a)

(b)

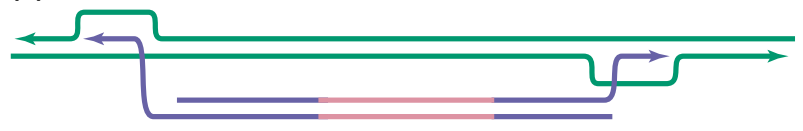

(c)

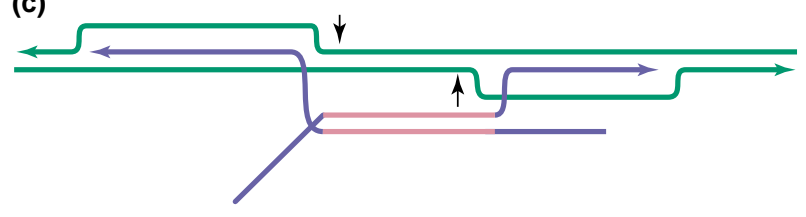

(d)

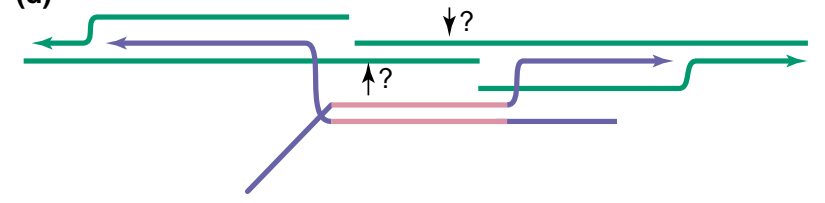

(e)

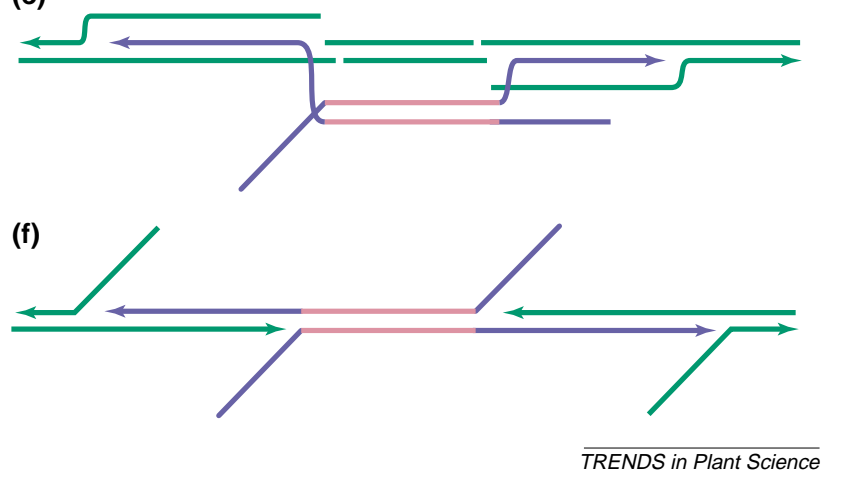

Fig. 2. A model for gene replacement in mammals [43]. (a) Resection of transgene ends. (b) RAD51-driven homology search. (c) Branch migration ends at the heterology-homology border. (d) ERCC1/XPF-dependent incision of the target site just $5^{\prime}$ of the displaced $3^{\prime}$ strand. (e) Nuclease or helicase activity eliminates the targeted DNA segment. (f) DNA synthesis, a $5^{\prime}$ flap endonuclease (FEN1) and DNA ligase complete the gene-replacement process. Arrows indicate canonical ERCC1/XPF recognition sites [43]. The question marks indicate more questionable sites; sites that might or might not be cleaved by ERCC1/XPF. Arrowheads represent $3^{\prime}$ ends; the invading (damaged, or transgene) strands are depicted in purple; the (homologous) template strands are in green. The pink insert represents an insertion (such as a selectable marker) with no homology to the target.

fidelity of repair of a targeted (I-SceI-induced) break in tobacco lines overexpressing a nuclear-targeted RecA was characterized [51], and the frequency at which breaks were repaired entirely through HR was found to have increased. Interestingly, however, the frequency of gene replacement events did not increase.

Overexpression of RAD51 and/or RAD52 has also been investigated in mammalian cell lines, and has produced conflicting results, including an increase in gene conversion at a targeted break [52], a decrease in HR repair of a similar site [53], and an increase in spontaneous HR. It is possible that an excessive level of overexpression of RAD51 inhibits repair of DSBs by HR.

\section{Nonhomologous end joining: a target for elimination} Our understanding of the nonhomologous end-joining pathway is largely derived from research in vertebrate 
cell lines, confirmed by experiments in $S$. cerevisiae employing a HR-defective (rad52) background. Mammalian mutations affecting NHEJ and ionizing radiation (IR) sensitivity include cell lines defective in Ku80 and Ku70 (DSB binding proteins), DNA ligase IV, and its cofactor, XRCC4 [54]. Genetic evidence in S. cerevisiae, as well as recent in vitro evidence, indicate that the MRE11/RAD50/XRS2 complex also plays an essential role in NHEJ, perhaps in maintaining the alignment of ends [55-57]. The Arabidopsis genome encodes obvious homologs of Ku70, Ku80, Lig4, XRCC4, MRE11 and RAD50. In vertebrates, $\mathrm{DNA}-\mathrm{PK}_{\mathrm{cs}}$ interacts with $\mathrm{Ku}$ to assist in bringing DSBs together. Neither Arabidopsis nor yeast encodes a DNA-PK $\mathrm{Cs}_{\mathrm{cs}}$ homolog, but they do encode homologs of the closely related proteins ATM [58] and ATR, which are involved in response to DNA-damaging agents. Arabidopsis mutants defective in $\mathrm{Ku} 70$ and ATM are sensitive to gamma radiation; other mutants have not yet been tested for IR sensitivity [59,60]. The Arabidopsis Ku80, Ku70, DNA ligase IV and RAD51 genes are transcriptionally induced in response to IR or bleomycin, suggesting that they play a role in DSB repair [60-62].

It is possible that the $\mathrm{HR}$ and NHEJ repair pathways not only coexist but also compete for DSBs. An end captured by $\mathrm{Ku}$ complex might become inaccessible to either RAD51 or the nucleases and accessory proteins required to prime the substrate. For this reason, elimination of the NHEJ pathway might not only enhance the ratio of homologous to nonhomologous transformation events, but might raise the absolute frequency of gene replacement events per introduced transgene. It will be interesting to determine whether lines with deficiencies in the early stages of NHEJ (Ku mutants) have enhanced frequencies of gene targeting.

\section{Oligonucleotide-directed gene targeting}

Self-complementary chimeric $\mathrm{DNA} / 2^{\prime}-O$-methyl RNA (chimeras) and modified DNA oligonucleotides direct site-specific base changes in chromosomal and episomal targets in mammalian cells [63-66]. Designed to pair with a homologous sequence within genomic DNA, these molecules introduce single base changes at specified targets (Fig. 3). Initial studies using mammalian culture cells were successful in correcting a targeted mutation in $\beta$-globin [64] and in an alkaline phosphatase gene [63]. Subsequent experiments in animals were directed toward restoring expression of mouse dystrophin [65] and canine muscle [66]. Gene correction efficiencies reported in these findings were in the 1 to $20 \%$ range.

A proposed mechanism by which these molecules act involves the DNA strand of the chimera functioning as a template for gene repair acted upon by the host DNA repair machinery while the RNA strand enhances targeting efficiency by stabilizing complex formation with the target DNA sequence [67].

The utility of chimeras to introduce site-specific base changes has also been demonstrated in plants. Chimeras were introduced into tobacco [68] and maize [69,70] to target endogenous and transgenes resulting in selectable

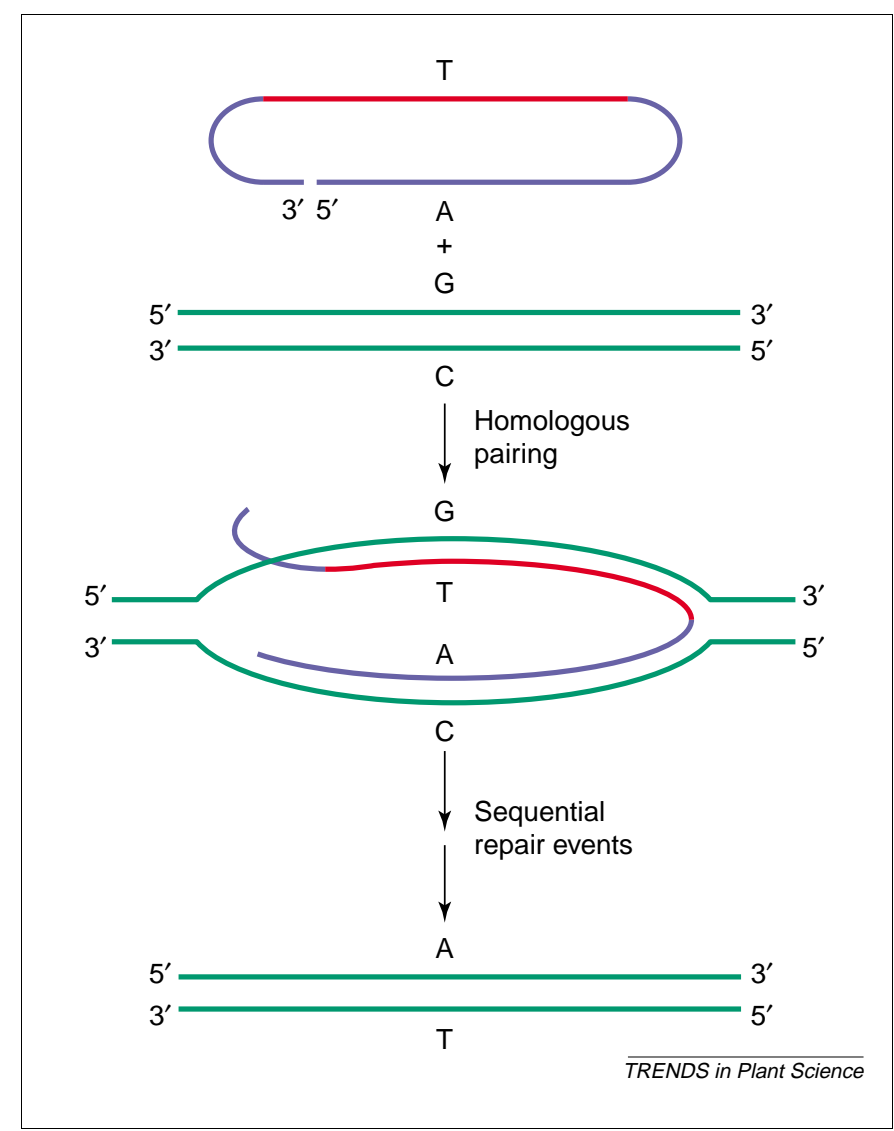

Fig. 3. Integration of a targeting oligonucleotide into double-stranded DNA $[68,72]$. The chimeric oligonucleotide (RNA: red, DNA: purple) interacts through homologous base paring with the target DNA (green) and results in the formation of a double D-loop structure. The mismatched base pairs are resolved through a series of DNA repair processes.

phenotypes. Unlike the high level of efficiencies observed in mammalian systems, oligonucleotide-directed gene conversion occurs at a low frequency $\left(10^{-4}\right)$ in plants [68,69].

Data from chimera-directed gene-targeting experiments in plants yielded results never before observed in other systems. Nonspecific conversions in planta were detected at the targeted base and at the position immediately $5^{\prime}$. Whether this in vivo lack of precision, or slippage, is limited to certain plant species is unknown. However, these 'ectopic' conversions are not observed in plasmid targeting experiments involving maize or Arabidopsis cell free extracts (CFE) and have only been observed in vitro when using tobacco CFEs [71].

To help elucidate the factors involved in the conversion mechanism and as a system to study plant DNA repair mechanisms, a cell-free assay utilizing protein extracts from either plastids or whole cells has been established [71,72]. This work has also provided a mechanistic explanation of the in vivo plant results. Chimeras and modified, single stranded DNA oligonucleotides containing phosphorothioate linkages can both convert or insert a targeted base within a plasmid DNA substrate [72].

Current examples of oligonucleotide-directed plant gene targeting were directed toward base changes 
resulting in a selectable phenotype. The current low level of targeting efficiency precludes the use of addressing genes yielding a non-selectable phenotype. Improvements in vector design and an increase in our knowledge of plant DNA repair mechanisms will expedite the applicability of this technology. Oligonucleotide-directed gene targeting provides us with one possible approach to target precise regions of the plant genome. It is unlikely that this technology will be applicable in efforts directed at the attenuation of plant gene expression. However, it should be useful for generating systematic amino acid substitutions or for targeting select members of complex gene families.

\section{Perspectives}

A milestone in the progression of plant biology and crop varietal improvement will be the ability to target readily and to modify specifically genes of interest in a wide range of plant species. The ability to make precise gene modifications in crop species might help to alleviate many of the concerns associated with the current biotechnological approaches that are integral to their improvement.

With the exception of the lower land plant Physcomitrella, homologous recombination remains an elusive tool for use in plant genetic engineering. Given limited successes in higher plant species, Physcomitrella might provide an excellent model system for determining the mechanisms by which homologous recombination and gene targeting occurs in plants [73]. The manipulation of the expression of genes involved in recombination might alter transgene integration pathways and make higher plants more Physcomitrella-like in this respect. In addition, the judicious use of counterselectable flanking markers (which kill plants that have integrated the transgene nonhomologously) has already been shown to bring transgene replacement to the verge of feasibility in rice [74] (see the Research Focus article by Barbara Hohn and Holger Puchta in this issue of Trends in Plant Science).

In efforts to use the vast amounts of genomic information, we will continue to rely upon insertional mutagenesis and RNAi approaches to assist us in assigning function to newly discovered plant genes. The processes of homology-directed transgene integration and in vivo site-specific gene changes through the use of chimeric and modified-DNA oligonucleotides are currently too inefficient to compete with the less precise, but more accessible techniques of RNAi and random insertional mutagenesis.

It is much too early to predict whether more precise genetic engineering techniques will ever become a readily accessible technology in all higher plants. However, the growing interest and participation in this field and the rate at which plant DNA repair and gene targeting discoveries are being made is an encouraging and essential step in the right direction.

\section{Acknowledgements}

Research performed by A.B.B.'s laboratory in the area of DNA doublestrand-break repair was funded by the National Science Foundation, the
USDA and BARD. Oligonucleotide-directed gene-targeting efforts in G.D.M.'s laboratory was supported by the Samuel Roberts Noble Foundation.

\section{References}

1 Krysan, P.J. et al. (1999) T-DNA as an insertional mutagen in Arabidopsis. Plant Cell 11, 2283-2290

2 Meyer, P. and Saedler, H. (1996) Homology-dependent gene silencing in plants. Annu. Rev. Plant Physiol. Plant Mol. Biol. 47, 23-48

3 Fire, A. (1999) RNA-triggered gene silencing. Trends Genet. 15, $358-363$

4 Zamore, P.D. et al. (2000) RNAi:double-stranded RNA directs the ATP-dependent cleavage of mRNA at 21 to 23 nucleotide intervals. Cell 101, 25-33

5 Kooter, J.M. et al. (1999) Listening to the silent genes: transgene silencing, gene regulation and pathogen control. Trends Plant Sci. 4, $340-347$

6 Vance, V. and Vaucheret, H. (2001) RNA silencing in plants - defense and counterdefense. Science 292, 2277-2280

7 Waterhouse, P.M. et al. (2001) Role of short RNAs in gene silencing. Trends Plant Sci. 6, 297-301

8 Chuang, C-F. and Meyerowitz, E.M. (2000) Specific and heritable genetic interference by double-stranded RNA in Arabidopsis thaliana. Proc. Natl. Acad. Sci. U. S. A. 97, 4985-4990

9 Smith, N. et al. (2000) Total silencing by intron-spliced hairpin RNAs. Nature 407, 319-320

10 Kumagai, M.H. et al. (1995) Cytoplasmic inhibition of carotenoid biosynthesis with virus-derived RNA. Proc. Natl. Acad. Sci. U. S. A. 92, 1679-1683

11 Ruis, M.T. et al. (1998) Initiation and maintenance of virus-induced gene silencing. Plant Cell 10, 937-946

12 Bundock, P. et al. (1995) Trans-kingdom T-DNA transfer from A. tumefaciens to $S$. cerevisiae. EMBO J. 14, 3206-3214

13 Piers, K. et al. (1996) Agrobacterium tumefaciens-mediated transformation of yeast. Proc. Natl. Acad. Sci. U. S. A. 93, 1613-1618

14 Kunik, T. et al. (2001) Genetic transformation of HeLa cells by Agrobacterium. Proc. Natl. Acad. Sci. U. S. A. 98, 1871-1876

15 te Riele, H. et al. (1992) Highly efficient gene targeting in embryonic stem cells through homologous recombination with isogenic DNA constructs. Proc. Natl. Acad. Sci. U. S. A. 89, 5128-5132

16 Sonoda, E. et al. (2001) Homologous DNA recombination in vertebrate cells. Proc. Natl. Acad. Sci. U. S. A. 98, 8388-8394

17 Schaefer, D.G. et al. (1994) Genetic transformation of the moss Physcomitrella patens. In Biotechnology in Agriculture and Forestry (Bajaj, Y.P.S. et al., eds), pp. 349-364, Springer Verlag

18 Kammer, W. and Cove, D.J. (1996) Genetic analysis of the result of retransfomation of transgenic lines of the moss, Physcomitrella patens. Mol. Gen. Genet. 20, 380-382

19 Schaefer, G. (2001) Gene targeting in Physcomitrella patens. Curr. Opin. Plant Biol. 4, 143-150

20 Puchta, H. (1999) Double-strand break-induced recombination between ectopic homologous sequences in somatic plant cells. Genetics $152,1173-1181$

21 Jasin, M. (2000) Homology-directed repair in mammalian cells. Environ. Mol. Mutagen. 35 (Suppl. 31), 32

22 Liang, F. et al. (1998) Homology-directed repair is a major doublestrand break repair pathway in mammalian cells. Proc. Natl. Acad. Sci. U. S. A. 95, 5172-5177

23 Siebert, R. and Puchta, H. (2002) Efficient repair of genomic double-strand breaks by homologous recombination between directly repeated sequences in the plant genome. Plant Cell 14, 1121-1131

24 Reiss, B. et al. (2000) RecA stimulates sister chromatid exchange and the fidelity of double-strand break repair, but not gene targeting, in plants transformed by Agrobacterium. Proc. Natl. Acad. Sci. U. S. A. 97, 3358-3363

25 Kempin, S. et al. (1997) Targeted disruption in Arabidopsis. Nature 389, 802-803

26 Miao, Z-H. and Lam, E. (1995) Targeted disruption of the TGA3 locus in Arabidopsis thaliana. Plant J. 7, 359-365

27 The Arabidopsis Genome Initiative, (2000) Analysis of the genome 
sequence of the flowering plant Arabidopsis thaliana. Nature 408, $796-815$

28 Jenkins, M.E. et al. (1995) Radiation-sensitive mutants of Arabidopsis thaliana. Genetics 140, 725-732

29 Harlow, G.R. et al. (1994) Isolation of uvh1, an Arabidopsis mutant hypersensitive to ultraviolet light and ionizing radiation. Plant Cell 6, $227-235$

30 Jiang, C.Z. et al. (1997) UV- and gamma-radiation sensitive mutants of Arabidopsis thaliana. Genetics 147, 1401-1409

31 Masson, J. et al. (1997) Mutants of Arabidopsis thaliana hypersensitive to DNA damaging treatments. Genetics 146, 401-407

32 Masson, J. and Paszkowski, J. (1997) Arabidopsis thaliana mutants altered in homologous recombination. Proc. Natl. Acad. Sci. U. S. A. 94, 11731-11735

33 Hefner, E. et al. Arabidopsis mutants sensitive to gamma radiation include the homolog of the human repair gene ERCC1. J. Exp. Bot. (in press)

34 Tamura, K. et al. (2002) Identification of Ku70 and Ku80 homologues in Arabidopsis thaliana: evidence for a role in the repair of DNA double-strand breaks. Plant J. 29, 771-781

35 Nam, J. et al. (1999) Identification of T-DNA tagged Arabidopsis mutants that are resistant to transformation by Agrobacterium. Mol. Gen. Genet. 261, 429-438

36 Mysore, K.S. et al. (2000) An Arabidopsis histone H2A mutant is deficient in Agrobacterium T-DNA integration. Proc. Natl. Acad. Sci. U. S. A. $97,948-953$

37 Gelvin, S.B. (2000) Agrobacterium and plant genes involved in T-DNA transfer and integration. Annu. Rev. Plant. Physiol. Plant Mol. Biol. $51,223-256$

38 Paques, F. and Haber, J.E. (1999) Multiple pathways of recombination induced by double-strand breaks in S. cerevisiae. Micro. Mol. Biol. Rev. 63, 349-404

39 Puchta, H. (1998) Repair of genomic double-strand breaks in somatic plant cells by one-sided invasion of homologous sequences. Plant J. 13, 331-339

40 Rubin, E. and Levy, A.A. (1997) Abortive gap repair: underlying mechanism for $D s$ element formation. Mol. Cell. Biol. 17, 6294-6302

41 Rong, Y.S. and Golic, K.G. (2001) A targeted gene knockout in Drosophila. Genetics 157, 1307-1312

42 Niedernhofer, L.J. et al. (2001) The structure-specific endonuclease Ercc1-Xpf is required for targeted gene replacement in embryonic stem cells. $E M B O$ J. 20, 6540-6549

43 de Laat, W.L. et al. (1998) DNA structural elements required for ERCC1-XPF endonuclease activity. J. Biol. Chem. 273, 7835-7842

44 Dubest, S. et al. (2002) Role of the AtRad1p endonuclease in homologous recombination in plants. EMBO Rep. 3, 1049-1054

45 Fidantsef, A. et al. (2000) The Arabidopsis UVH1 gene is a homolog of the yeast repair endonuclease RAD1. Plant Physiol. $124,579-586$

$46 \mathrm{Liu}, \mathrm{Z}$. et al. (2000) Repair of UV damage in plants by nucleotide excision repair: Arabidopsis UVH1 DNA repair gene is a homolog of Saccharomyces cerevisiae Rad1. Plant J. 21, 519-528

47 Nam, J. et al. (1998) Agrobacterium tumefaciens transformation of the radiation hypersensitive Arabidopsis thaliana mutants uvh1 and rad5. Mol. Plant-Microbe Interact. 11, 1136-1141

48 Preuss, S. et al. (1999) Radiation-sensitive Arabidopsis mutants are proficient for T-DNA transformation. Mol. Gen. Genet. 261, $623-626$

49 Sonti, R.V. et al. (1995) Arabidopsis mutants deficient in T-DNA integration. Proc. Natl. Acad. Sci. U. S. A. 92, 11786-11790

50 Reiss, B. et al. (1996) RecA protein stimulates homologous recombination in plants. Proc. Natl. Acad. Sci. U. S. A. 93, 3094-3098

51 Shalev, G. et al. (1999) Stimulation of homologous recombination in plants by expression of the bacterial resolvase RuvC. Proc. Natl. Acad. Sci. U. S. A. 96, 7398-7402

52 Lambert, S. and Lopez, B.S. (2000) Characterization of mammalian RAD51 double strand break repair using non-lethal dominantnegative forms. EMBO J. 19, 3090-3099

53 Kim, P.M. et al. (2001) Overexpression of human RAD51 and RAD52 reduces double-strand break-induced homologous recombination in mammalian cells. Nucleic Acids Res. 29, 4352-4360
54 van Gent, D.C. et al. (2001) Chromosomal stability and the DNA double-stranded break connection. Nat. Rev. Genet. 2, 196-206

55 Lewis, L.K. et al. (2002) Differential suppression of DNA repair deficiencies of yeast rad50, mre11 and xrs2 mutants by EXO1 and TLC1 (the RNA component of telomerase). Genetics 160, 49-62

56 Huang, J. and Dynan, W.S. (2002) Reconstitution of the mammalian DNA double-strand break end-joining reaction reveals a requirement for an Mre11/Rad50/NBS1-containing fraction. Nucleic Acids Res. 30, $667-674$

57 Chen, L. et al. (2001) Promotion of Dnl4-catalyzed DNA end-joining by the Rad50/Mre11/Xrs2 and Hdf1/Hdf2 complexes. Mol. Cell 8, $1105-1115$

58 Garcia, V. et al. (2000) An ATM homologue from Arabidopsis thaliana: complete genomic organisation and expression analysis. Nucleic Acids Res. 28, 1692-1699

59 Riha, K. et al. (2002) Telomere length deregulation and enhanced sensitivity to genotoxic stress in Arabidopsis mutants deficient in Ku70. EMBO J. 21, 2819-2826

60 Valérie, G. et al. AtATM is essential for meiosis and the somatic response to DNA damage in plants. Plant Cell (in press)

61 West, C.E. et al. (2000) Arabidopsis DNA ligase IV is induced by gamma-irradiation and interacts with an Arabidopsis homologue of the double strand break repair protein XRCC4. Plant J. 24, $67-78$

62 Tamura, K. et al. (2002) Identification of Ku70 and Ku80 homologues in Arabidopsis thaliana: evidence for a role in the repair of DNA double-strand breaks. Plant J. 29, 771-781

63 Yoon, K. et al. (1996) Targeted gene correction of episomal DNA in mammalian cells mediated by a chimeric RNA/DNA oligonucleotide. Proc. Natl. Acad. Sci. U. S. A. 93, 2071-2076

64 Cole-Strauss, A. et al. (1996) Correction of the mutation responsible for sickle cell anemia by an RNA/DNA oligonucleotide. Science 273, $1386-1389$

65 Rando, T.A. et al. (2000) Rescue of dystrophin expression in mdx mouse muscle by RNA/DNA oligonucleotides. Proc. Natl. Acad. Sci. U. S. A. 97, 5363-5368

66 Bartlett, R.J. et al. (2000) In vivo targeted repair of a point mutation in the canine dystrophin gene by a chimeric RNA/DNA oligonucleotide. Nat. Biotechnol. 18, 615-622

67 Gamper, H.B. et al. (2000) A plausible mechanism for gene correction by chimeric oligonucleotides. Biochemistry $39,5808-5816$

68 Beetham, P.R. et al. (1999) A tool for functional plant genomics: cChimeric RNA/DNA oligonucleotides cause in vivo gene-specific mutations. Proc. Natl. Acad. Sci. U. S. A. 96, 8774-8778

$69 \mathrm{Zhu}$, T. et al. (1999) Targeted manipulation of maize genes in vivo using chimeric RNA/DNA oligonucleotides. Proc. Natl. Acad. Sci. U. S. A. 96, 8768-8773

$70 \mathrm{Zhu}$, T. et al. (2000) Engineering herbicide-resistant maize using chimeric RNA/DNA oligonucleotides. Nat. Biotechnol. 18, $555-558$

71 Rice, M.C. et al. (2000) Genetic repair of mutations in plant cell-free extracts directed by specific chimeric oligonucleotides. Plant Physiol. 123, 427-437

72 Kmiec, E.B. et al. (2001) Chloroplast lysates support directed mutagenesis via modified DNA and chimeric RNA/DNA oligonucleotides. Plant J. 27, 267-274

73 Schaefer, D.G. (2002) A new moss genetics: targeting mutagenesis in Physcomitrella patens. Annu. Rev. Plant Biol. 53, 477-501

74 Terada, R. et al. (2002) Efficient gene targeting by homologous recombination in rice. Nat. Biotechnol. 20, 1030-1034

75 Constantinou, A.D. et al. (2001) Branch migration and Holliday junction resolution catalyzed by activities from mammalian cells. Cell $104,259-268$

76 Gallego, M.E. and White, C.I. (2001) RAD50 function is essential for telomere maintenance in Arabidopsis. Proc. Natl. Acad. Sci. U. S. A. 98, 1711-1716

77 Gerbi, H. et al. (2001) Homologous recombination in planta is stimulated in the absence of Rad50. EMBO Rep. 21, 287-291

78 Gallego, M.E. et al. (2001) Disruption of the Arabidopsis RAD50 gene leads to plant sterility and MMS sensitivity. Plant J. 25, $31-41$ 